Supporting Information

\title{
Preparation of butadiene-bridged \\ polymethylsiloxane/ethylcellulose/1-carboxymethyl-3-methylimidazolium chloride ternary composite membranes for gas separation
}

Wenqiang $\mathrm{Ma}^{1}$, Shuangping $\mathrm{Xu}^{1, *}$, Hongge $\mathrm{Jia}^{1, *}$, Jingyu $\mathrm{Xu}^{1,2, *}$, Da Liu ${ }^{3}$, Mingyu

Zhang ${ }^{1}$, Yanqing $\mathrm{Qu}^{1}$, Hailiang Zhou ${ }^{1}$, Yushu Zhang ${ }^{1}$, Xintian Wang ${ }^{1}$, Wenwen Zhao $^{1}$

${ }^{1}$ College of Materials Science and Engineering, Heilongjiang Provinces Key Laboratory of Polymeric Composite materials, Qiqihar University, Qiqihar, 161006, China.

${ }^{2}$ Liaoning Key Lab of Lignocellulose Chemistry and BioMaterials, Liaoning Collaborative Innovation Center for Lignocellulosic Biorefinery, College of Light Industry and Chemical Engineering, Dalian Polytechnic University, Dalian 116034, China.

${ }^{3}$ College of Chemical Engineering, Daqing Normal University, Daqing, 163712, China.

* Authors to whom any correspondence should be addressed. 
E-mail: 02228@qqhru.edu.cn(X. S.), jiahongge@qqhru.edu.cn(J. H.), xjy951011@163.com (J. X.).

\section{Calculations}

The pure gas permeance $P$ by the following relation (1):

$$
P_{g}=\left(\frac{d p}{d t}\right) \frac{V \cdot T_{0}}{A \cdot \Delta p \cdot P_{0} \cdot \mathrm{T}}\left(\mathrm{ml} \bullet \mathrm{cm} \bullet \mathrm{cm}^{-2} \bullet \mathrm{s}^{-1} \bullet \mathrm{cmHg}^{-1}\right)
$$

Where, $d p / d t$ is the slope $(\mathrm{cmHg} / \mathrm{s})$ of the pressure against time in the pseudo-steady-state, $V$ is the downstream volume $\left(\mathrm{cm}^{3}\right)$ of gas permeation, $\Delta p(\mathrm{cmHg})$ is the difference in the pressure between the two sides of the membrane, $A$ is the effective membrane area $\left(3.14 \mathrm{~cm}^{2}\right), P_{0}$ is $76 \mathrm{cmHg}, T_{0}$ is $273 \mathrm{~K}$, and $T$ is the measured temperature of $307 \mathrm{~K}$.

The mixed gas permeability coefficient $P$ was calculated by the following, Equation (2)

$$
P=\frac{\mathrm{q} \times K \times \mathrm{L}}{\mathrm{a} \times \mathrm{p} \times \mathrm{t}}\left(\mathrm{ml} \bullet \mathrm{cm} \bullet \mathrm{cm}^{-2} \bullet \mathrm{s}^{-1} \bullet \mathrm{cmHg}^{-1}\right)
$$

Where, $\mathrm{q}$ is transmission volume $(\mathrm{mL}) ; \mathrm{K}$ is auxiliary positive coefficient (the fixed value is 2), meaning that it is the setting point instrument by factory; $\mathrm{L}$ is film thickness $(\mathrm{cm})$; $\mathrm{p}$ is permeability pressure $(\mathrm{cmHg})$; $\mathrm{t}$ is measurement time $(\mathrm{s})$; and $\mathrm{a}$ is the area of the gas permeation film (the fixed value is $0.785 \mathrm{~cm}^{2}$ ).

In this experiment, the gas separation factor was calculated by the following relation (3) and relation (4):

$$
\alpha=\frac{P_{\mathrm{O}_{2}}}{P_{N_{2}}}
$$




$$
\alpha=\frac{P_{\mathrm{CO}_{2}}}{P_{N_{2}}}
$$

Where $\mathrm{PO}_{2}, P \mathrm{PO}_{2}$ and $P \mathrm{~N}_{2}$ correspond to single gas $\mathrm{O}_{2}, \mathrm{CO}_{2}$ and $\mathrm{N}_{2}$, them can be calculated from equation (1) and where $P_{2}, P_{C O}$ and $P \mathrm{~N}_{2}$ correspond to mixed gas $\mathrm{O}_{2}, \mathrm{CO}_{2}$ and $\mathrm{N}_{2}$, them can be calculated from equation (2).

The diffusion coefficients (D) and the solubility coefficients (S) were calculated by the following equations:

$$
\begin{aligned}
& D=\frac{L^{2}}{6 T} \\
& S=\frac{P}{D}
\end{aligned}
$$

Where $\mathrm{L}$ and $\mathrm{T}$ are the amount of the thickness of the membrane and the time lag.

\begin{tabular}{|c|c|c|c|c|c|c|c|c|}
\hline \multirow{2}{*}{ Sample } & \multicolumn{2}{|c|}{$\mathrm{GPC}^{\text {a) }}$} & \multicolumn{5}{|c|}{ Solubility b) } & \multirow{2}{*}{$\begin{array}{l}\text { Membrane } \\
\text { forming ability }\end{array}$} \\
\hline & $M n$ & $M w / M n$ & $\mathrm{CHCl}_{3}$ & THF & DMF & NMP & methanol & \\
\hline BBPMS & $3.19 \times 10^{4}$ & 1.97 & + & + & + & + & - & Poor \\
\hline
\end{tabular}

\section{The molecular weight of BBPMS}

Table S1 Properties of BBPMS.

a) THF, polystyrenestandard; b) + : soluble, -: insoluble.

3. The small scale of SEM images of EC/IL membrane and BBPMS/EC/IL mixed matrix membranes 


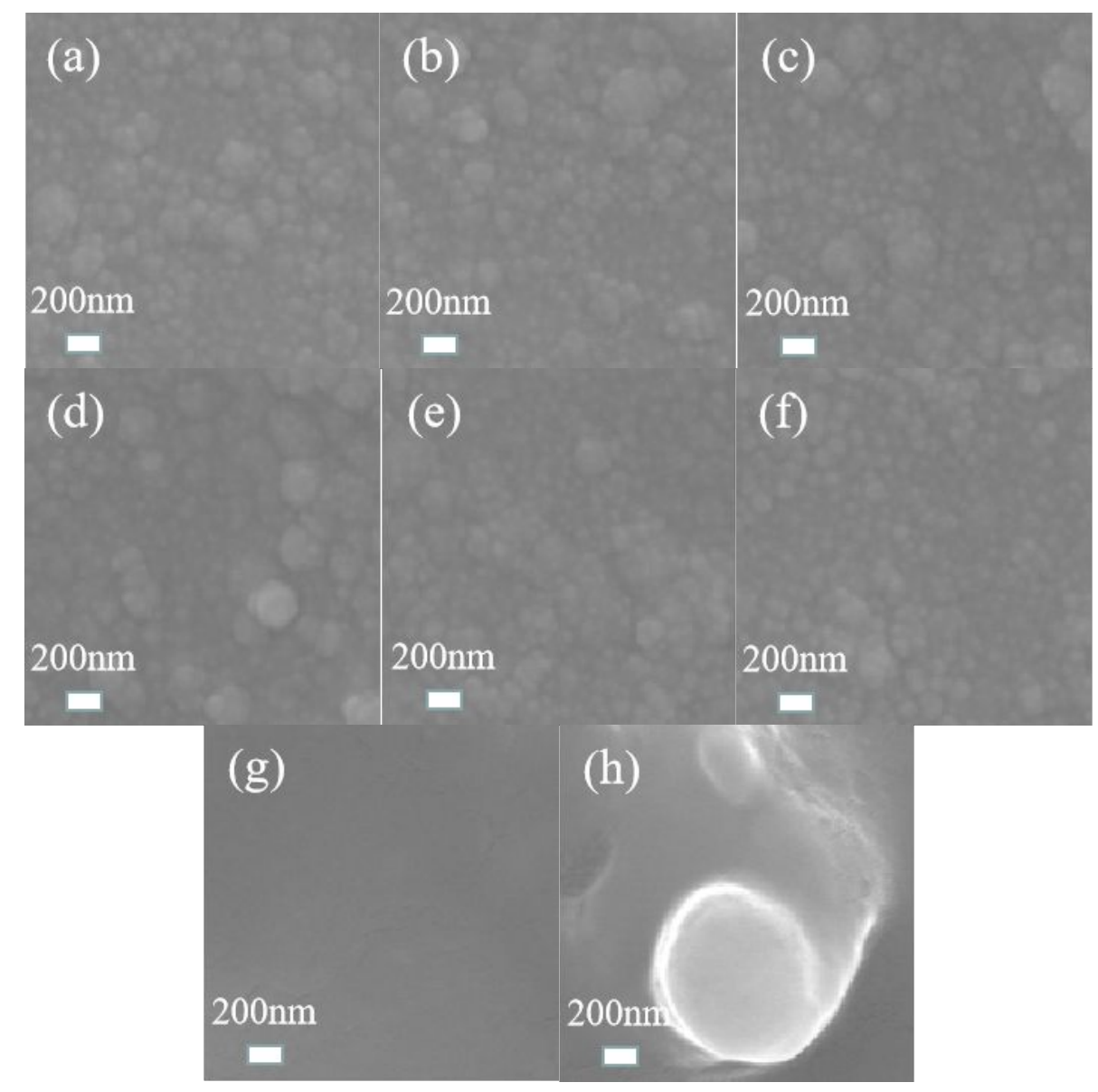

Figure S1 The small scale of SEM images of EC/IL membrane (g) and BBPMS/EC/IL mixed matrix membranes (a: $\operatorname{MMM(90\% ),~b:~} \operatorname{MMM(80\% ),~c:~} \operatorname{MMM(70\% ),~d:~} \operatorname{MMM(60\% ),~e:~}$ MMM(50\%), f: MMM(40\%), h: BBPMS)

4. The contribution of the BBMPS structure itself to the improvement of permeability

The peak located at $2165 \mathrm{~cm}^{-1}$ represents the asymmetrical stretching vibration of $\mathrm{Si}-\mathrm{H}$ from BBPMS, $\mathrm{CO}_{2}$ can adsorbed on thesurface of silicon and inserted into $\mathrm{Si}-\mathrm{H}$ bonds to obtained $\mathrm{Si}-\mathrm{O}-\mathrm{C}(=\mathrm{O})-\mathrm{H}[\mathrm{S} 1]$. After the gas has passed through, the $\mathrm{Si}-\mathrm{H}$ surface can be regenerated, and then this reaction was continued [S2]. The mechanism of permselectivity is mainly based on the small size difference 
(molecular-sieving effects) between oxygen $(0.34 \mathrm{~nm})$ and nitrogen $(0.36 \mathrm{~nm})$ [S3]. Finally, framework regenerate and the gas passes smoothly. Furthermore, BBPMS/EC hybrid membranes will provide a large-specific-area $\mathrm{Si}-\mathrm{H}$ surface, accelerating the $\mathrm{CO}_{2}$ and $\mathrm{O}_{2}$ fixing reaction [S2]. The peak located at $1261 \mathrm{~cm}^{-1}$ represents the symmetric bending vibration of $\mathrm{Si}-\mathrm{CH}_{3}$ from BBPMS, the alkyl functional groups may provide the new sites for the material to adsorb gas molecules [S4].

5. The TG analysis of EC/IL membrane and BBPMS/EC/IL membranes $\left(30-100^{\circ} \mathrm{C}\right)$

As can be seen from Figure S2, there is almost no weight loss at temperatures between $30-100^{\circ} \mathrm{C}$.

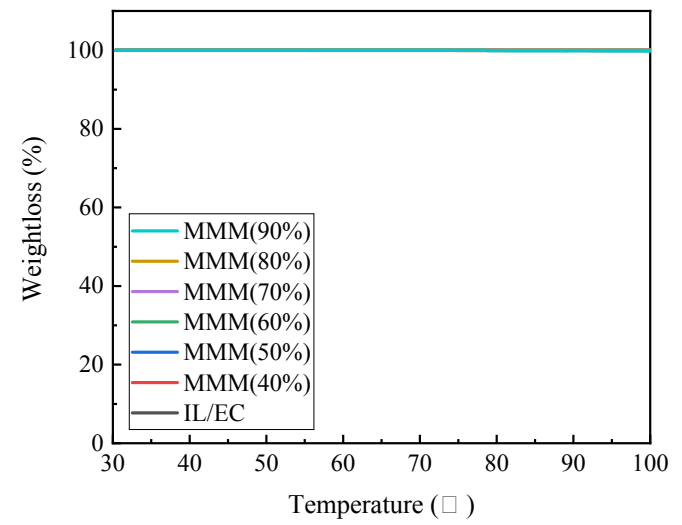

Figure S2 TG analysis of EC/IL membrane and BBPMS/EC/IL membranes $\left(30-100^{\circ} \mathrm{C}\right)$

6. BET surface area measurements of IL/EC, MMMs and BBPMS complex

Table S2 BET surface area measurements of IL/EC, MMMs and BBPMS complex membrane.

\begin{tabular}{cccc}
\hline Sample & BET Surface $\left(\mathrm{m}^{2} / \mathrm{g}\right)$ & Pore Volume $\left(\mathrm{cm}^{3} / \mathrm{g}\right)$ & Pore Width $(\AA)$ \\
\hline IL/EC & 0.09 & $0.91 \times 10^{-4}$ & 3.68
\end{tabular}




\begin{tabular}{cccc}
$\operatorname{MMM}(40 \%)$ & 1.69 & $2.82 \times 10^{-4}$ & 5.35 \\
$\operatorname{MMM}(50 \%)$ & 1.77 & $3.23 \times 10^{-4}$ & 5.76 \\
$\operatorname{MMM}(60 \%)$ & 1.85 & $3.44 \times 10^{-4}$ & 6.07 \\
$\operatorname{MMM}(70 \%)$ & 2.60 & $5.83 \times 10^{-4}$ & 6.62 \\
$\operatorname{MMM}(80 \%)$ & 3.51 & $6.49 \times 10^{-4}$ & 7.13 \\
$\operatorname{MMM}(90 \%)$ & 3.92 & $6.51 \times 10^{-4}$ & 7.48 \\
BBPMS complex & & & 9.05 \\
membrane & 5.36 & $9.89 \times 10^{-4}$ & \\
\hline
\end{tabular}

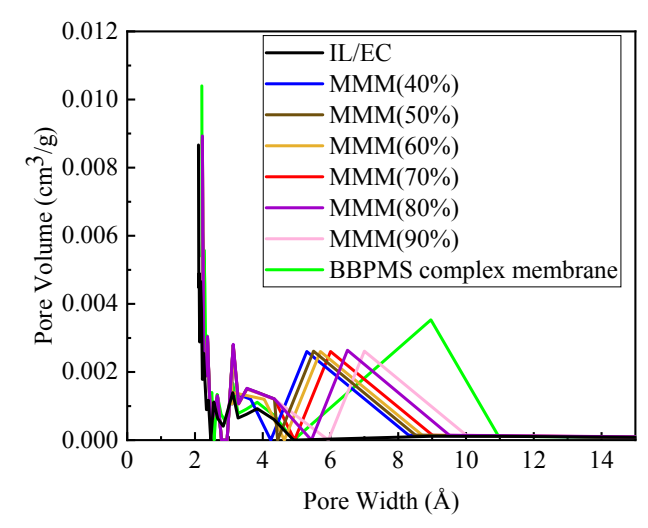

Figure S3 Pore size distribution of IL/EC, MMMs and BBPMS complex membrane.

\section{Refferences}

[S1] A. Schäfer, W. Saak, D. Haase, T. Müller, Silyl Cation Mediated Conversion of $\mathrm{CO}_{2}$ into Benzoic Acid, Formic Acid, and Methanol, Angewandte Chemie International Edition, 2012, 51, 2981-2984.

https://doi.org/10.1002/anie.201107958

[S2] Z. Fan, F. Liao, H. Shi, One-Step Direct Fixation of Atmospheric $\mathrm{CO}_{2}$ by Si-H 
Surface in Solution, iScience, 2019, 23, 100806.

https://doi.org/10.1016/j.isci.2019.100806

[S3] Y. Zang, T. Aoki, M. Teraguchi, New Synthetic Methods of Novel Nanoporous

Polycondensates and Excellent Oxygen Permselectivity of Their Composite Membranes, Nanomaterials, 2019, 9, 859.

https://doi.org/10.3390/nano9060859

[S4] G. Zhou, Z. Du, Y. Ma, Molecular simulation study on gas adsorption and separation performance of alkyl-functionalized HKUST materials, Computational Materials Science, 2020, 181, 109755.

https://doi.org/10.1016/j.commatsci.2020.109755
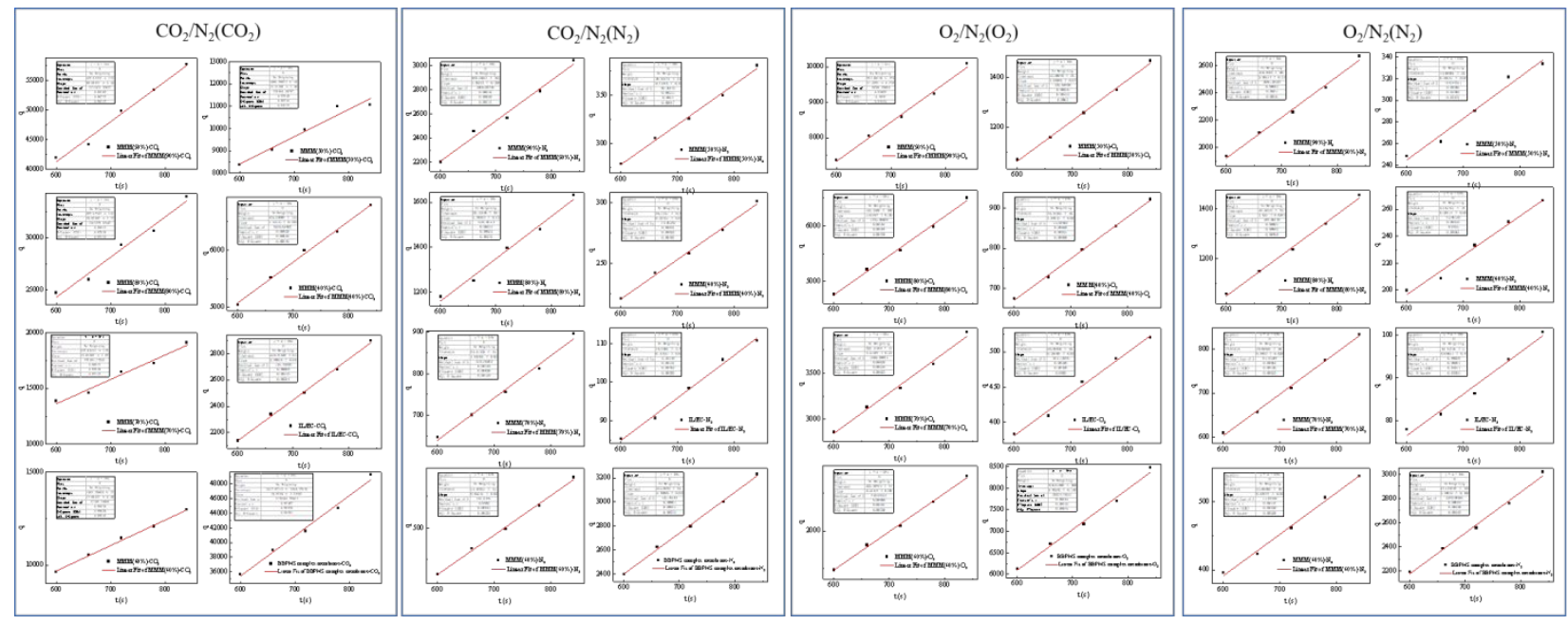

Figure S4 The curves of time-lag 